\title{
LA FEMINIZACIÓN DE LA POBREZA EN ESPAÑA: UN ENFOQUE DESDE LA PERSPECTIVA DE GÉNERO
}

\author{
THE FEMINIZATION OF POVERTY IN SPAIN: A GENDER PERSPECTIVE
}

\author{
SORAYA ESPINO GARCIA*1 \\ *UNIVERSIDAD DE CóRDOBa (ESPAÑA)
}

\section{RESUMEN}

El análisis de la pobreza en perspectiva de género en España resulta cada vez más necesario. Por ello, uno de los objetivos fundamentales es el de identificar, estudiar y analizar los factores de género que implican un diferencial de pobreza entre géneros en España, en detrimento de la mujer. Esta investigación analiza el acceso de la mujer al mercado de trabajo y cuál es la brecha de género tanto en el acceso al mercado laboral como en la participación en el mismo. La metodología utilizada en esta investigación ha sido la realización de un análisis cuantitativo utilizando los datos de la Encuesta de Población Activa (EPA) del Instituto Nacional de Estadística (INE) en el periodo temporal 2012-2021. Los principales resultados de la investigación muestran cómo la brecha por género es mucho más acusada en los periodos en los cuáles hay una crisis económica y la dificultad que tienen las mujeres para consolidarse plenamente en el mercado laboral en España, lo cual está llevando a un proceso continuo de feminización de la pobreza en España.

Palabras clave: Feminización de la pobreza, igualdad de género, desigualdad laboral, vulnerabilidad, mujeres.

\section{ABSTRACT}

The analysis of poverty from a gender perspective in Spain is necessary. Therefore, one of the fundamental objectives is to identify, study and analyze gender factors, which are linked to the gender economic gap in Spain. This research analyses women's access to the world of work and what is the gender gap in both, the access to the world of work and women's participation on it. The methodology used in this research had quantitative nature since the analysis have been implemented by making use of the data of the Active Population Survey (EPA in Spanish) of the National Statistics Institute (NSI) in a span of time which comprises the years from 2012 to 2021. The main results of the research show how the gender gap is much more acute in periods in which there is an economic crisis. It also shows the difficulty that women have to fully consolidate their professional careers in Spain. This process is narrowly linked to the feminization of poverty in Spain.

Keywords: Feminization of poverty, gender equality, labor inequality, vulnerability, women.

${ }_{1}^{1}$ Autor de correspondencia: Soraya Espino García.d02esgas@uco.es 


\section{Introducción}

Los datos actuales sobre personas que viven en situaciones de riesgo de pobreza y de exclusión social en España siguen mostrando cómo las mujeres se encuentran, cada vez más, en peores condiciones que los hombres. Así, muchas mujeres se siguen enfrentando, hoy en día, a barreras de diferente índole, como la formación, el cuidado de personas dependientes, etc., que la sociedad identifica con su género. Por tanto, estos factores aíslan e incluso marginan a la mujer. La pobreza aumenta la brecha de género y la desigualdad de género provoca, a su vez, pobreza. En consecuencia, nos encontramos ante dos desigualdades interconectadas que vulneran derechos de las mujeres.

El sistema patriarcal y la subsistencia de los roles de género provocan desigualdades sociales, culturales y económicas que generan dicha pobreza. La desigualdad laboral es uno de los principales factores que potencian la feminización de la pobreza con salarios más bajos, trabajos peores remunerados, con recurso a la economía sumergida, así como una mayor dedicación a los cuidados familiares. De hecho, el cuidado de los hijos y de personas dependientes sigue siendo un trabajo muy poco valorado y apenas reconocido por la sociedad, a pesar de que su reconocimiento debe de ser fundamental en el país avanzado en el que vivimos. A ello se añade que la crisis generada por la pandemia no ha hecho más que agravar esta realidad ya que se ha generado más precariedad laboral y más sobrecarga de trabajo para la mujer en lo referente al cuidado familiar, con el consiguiente lastre a la hora de conciliar, las dificultades para teletrabajar o, incluso, para mantener y conservar su empleo.

La incorporación de la mujer al mercado de trabajo en España se inicia a finales de la década de los años 70 del siglo XX (Cebrián López y Llorente Heras, 2017). Así, a partir de finales del siglo XX las mujeres asumen cada vez más trabajos anteriormente reservados a los hombres, $y$ gracias al esfuerzo del sistema educativo español y a la incorporación masiva de la mujer a la universidad, se posibilita que la mujer entre en puestos de trabajo cada vez de mayor responsabilidad.

Las diferentes reformas laborales que se producen en España, pretenden dar respuesta a mercados laborales cada vez más cambiantes y donde es necesaria una mayor innovación (García-Vega et al., 2021), aunque en ocasiones ello también implica una gran brecha de género.

El mercado de trabajo tiene un impacto muy significativo tanto en el hombre como en la mujer, debido a que las actuales condiciones de trabajo implican una constante entrada y salida del propio mercado laboral (Gómez Rufián, 2018), situación que especialmente complica a las mujeres, especialmente en lo referente a su conciliación con la vida familiar. Así, la incorporación de la mujer al mercado laboral va a depender de su situación familiar (Iglesias Fernández y Llorente Heras, 2001), con lo cual las políticas laborales deben de tener en cuenta esta situación. Ello se debe a que las mujeres tienen un mayor problema para incorporarse al mercado de trabajo y, en este sentido, deberían de establecerse mecanismos que favoreciesen dicha incorporación (Aragón Gómez, 2018), sobre la base de la necesaria conciliación de la vida profesional y de la vida familiar.

La flexibilidad laboral aparece como consecuencia de la necesidad que tienen las empresas para adaptarse a los mercados laborales (Cortès-Franch y Artazcoz, 2021). Sin embargo, las diferentes formas de flexibilidad laboral que existen, como serían las jornadas laborales reducidas, la contratación temporal o la subcontratación, afectan más negativamente a las mujeres, implicando unos menores salarios y unas peores condiciones laborales (Cortès-Franch y Artazcoz, 2021).

La relación que existe entre el género, el nivel de formación y el mercado de trabajo en España es fundamental. De hecho, si nos centramos en los estudios de formación profesional, uno de los niveles formativos que mayor aceptación tiene por parte del mercado laboral, la brecha de género está muy presente (Moreno Márquez, 2021), ya que existe una gran diferencia entre los estudios que eligen las mujeres y los que eligen los hombres. Por tanto, debería de analizarse qué tipo de 
estudios de formación profesional están cursando las mujeres con la finalidad de mejorar el acceso al mercado laboral (Moreno Márquez, 2021) y crear mejores condiciones de trabajo.

La transformación digital, que se ha acelerado durante la pandemia, implica la necesidad de una formación específica para obtener una serie de competencias que permita a toda persona ser competitiva en este mercado (Choi, 2021), nivel de formación que en ocasiones presenta una fuerte brecha de género debido al tipo de formación que reciben mayoritariamente las mujeres.

El actual modelo productivo en España tiende hacia un trabajo que se desarrolla, cada vez más, en plataformas digitales. Esta nueva tipología de trabajo puede ser positiva para las mujeres ya que otorga autonomía y flexibilidad, con lo cual se puede compatibilizar, por ejemplo, con los cuidados de los hijos o de personas dependientes. Sin embargo, también en este tipo de empresas existe una fuerte brecha de género ya que las personas que trabajan en los puestos mejor remunerados de las compañías de plataforma son hombres, y generalmente las personas que perciben los ingresos más bajos en estas empresas son las mujeres (Dios-Murcia et al., 2021) y los inmigrantes.

En el modelo de sociedad actual, la mujer se encarga fundamentalmente de todas las actividades de cuidados, tanto de hijos como de personas dependientes, siendo por tanto un pilar fundamental para un correcto funcionamiento del Estado de Bienestar en España (Quintero Lima y Díaz Gorkinkiel, 2021), aunque generalmente estas actividades están muy poco reconocidas por la sociedad y en su mayoría, incluso origina la existencia de mercados de trabajo no regulados, lo cierto es que implica un problema para el acceso a mercados de trabajo regulados para la propia mujer.

Por otro lado, cada vez es más frecuente que la existencia de situaciones en las que es la propia mujer la que se ocupa en solitario del cuidado y manutención de sus propios hijos, lo que conocemos como familias monoparentales. Este tipo de familias, registran una mayor probabilidad de llegar a situaciones de pobreza (Pérez Corral y Moreno Mínguez, 2021) frente a los hogares biparentales y, especialmente, cuando acontecen crisis económicas como la Crisis de 2008 (Pérez Corral y Moreno Mínguez, 2021) o la actual crisis originada por la pandemia de la Covid-19.

Así, uno de los mayores problemas con los que se encuentran las mujeres para acceder al mercado de trabajo se encuentra en la conciliación de su vida familiar y laboral. De hecho, es necesario una participación equilibrada de los hombres y de las mujeres en la vida familiar (Domínguez-Olabide, 2021) con la finalidad de mejorar e incentivar la incorporación plena de la mujer al mundo laboral. Esta necesidad debe de tener el respaldo de la propia sociedad.

El mercado laboral se ha visto muy afectado por la pandemia de la Covid-19. La superación de esta pandemia implica también un nuevo modelo productivo, que aborde un mercado laboral en España sujeto a desafíos de carácter tecnológico (López Rodríguez y González Gómez, 2021) y donde la mujer puede encontrar una fuerte brecha de género para adaptarse al mismo, debido tanto al nivel formativo que tiene como a la dificultad para conciliar la vida familiar y la laboral.

La pandemia de la Covid-19 ha tenido un impacto mayor en el mercado de trabajo femenino que en el masculino, especialmente en el campo del sector servicios (Albanesi y Kim, 2021). Siguiendo a Reichelt et al. (2021) la pandemia ha provocado una mayor brecha en el mercado de trabajo de las mujeres debido a que ha afectado en mayor medida a sus puestos de trabajo. En este sentido, el teletrabajo ha causado un impacto negativo debido al hecho de compaginar su trabajo con las labores del hogar y con la atención y cuidado a los hijos y personas dependientes (Salido Cortés, 2021).

\section{Objetivos e hipótesis}

El análisis y el estudio conjunto de la pobreza en perspectiva de género en España resultan muy necesarios. El resultado de este análisis nos lleva a confirmar que desde la crisis económica de 2008 hasta la situación actual provocada por la pandemia de la Covid-19, la tasa de pobreza de las mujeres ha ido incrementándose de forma significativa, lo que nos lleva a afirmar que nos 
encontramos ante un proceso de consolidación de la pobreza de las mujeres en España. Ello implica que las mujeres son cada vez más pobres que los hombres, y, en consecuencia, es necesario comprender y entender el impacto diferencial y la identificación de los factores de dicho empobrecimiento en nuestro país, en detrimento de la mujer.

Los objetivos principales que orientan esta investigación son los siguientes:

a) La identificación de los factores que implican un diferencial de pobreza entre géneros en España, derivada de la incorporación (o no) de la mujer al mercado laboral.

b) La incidencia de dichos factores en la mayor o menor disposición de las mujeres a incorporarse al mundo laboral.

c) El análisis de cómo la no incorporación de la mujer al mercado laboral debido al cuidado de sus hijos y/o de personas dependientes implica una mayor pobreza para las mujeres.

\section{Metodología}

\subsection{Diseño empleado}

La metodología empleada en esta investigación es de tipo cuantitativa. Para ello se ha utilizado la Encuesta de Población Activa (EPA) del Instituto Nacional de Estadística (INE) para el periodo temporal 2012-2021. Concretamente, se ha utilizado la EPA correspondiente al tercer trimestre de cada uno de estos años para poder realizar una comparación homogénea temporal entre dichos datos.

Sobre la base de los datos de la EPA se han elegido tres tasas diferentes. En primer lugar, la tasa de actividad para diferenciar por género entre la población activa y la población inactiva. En segundo lugar, la tasa de paro por género para diferenciar la población desempleada. Y, en tercer lugar, la tasa que analiza por qué las personas se ocupan del cuidado de personas dependientes y de los hijos, también diferenciada por género.

\subsection{Procedimiento y análisis de datos}

El análisis de los datos se ha realizado utilizando una hoja de cálculo Excel con la finalidad de realizar comparaciones entre los diferentes datos. Para ello, se han utilizado los datos derivados de la EPA correspondiente al tercer trimestre de cada uno de los años analizados.

El análisis se ha centrado en realizar una comparación por género de la tasa de actividad y de la tasa de paro en el periodo temporal 2012-2021. Para realizar esta comparación temporal, se ha elegido la EPA correspondiente al tercer trimestre, es decir, la correspondiente a los meses de julio, agosto y septiembre de cada año, para que los datos sean homogéneos y comparables entre sí.

Asimismo, también se ha realizado un análisis en cuanto a la conciliación de la vida familiar y el mercado de trabajo, analizando la relación con el cuidado de personas dependientes y de hijos.

Un tercer análisis que se ha realizado ha consistido en realizar un estudio empírico donde se compare la tasa de actividad y la tasa de paro en el tercer trimestre de 2021 con el objetivo de determinar cuál hubiera sido la tasa de paro si la incorporación de la mujer al mercado laboral hubiese sido la misma que la del hombre. Es decir, si la tasa de actividad, y en consecuencia la población activa, hubiese sido la misma.

\section{Resultados}

\subsection{Análisis de la tasa de actividad. Periodo 2012-2021}

La tasa de actividad viene dada por la relación entre la población activa y la población en edad de trabajar. Esta tasa de actividad es fundamental para determinar el número de personas activas e inactivas en un determinado país. En la tabla 1 se muestra la tasa de actividad en España en los diez últimos años, correspondientes a los datos de la EPA del tercer trimestre de cada año. Se 
utiliza los datos del tercer trimestre para poder realizar una comparación entre dichos datos, correspondientes al mismo periodo temporal

En dicha tabla se muestra claramente como existe una brecha de género en cuanto a la incorporación a la mujer al mercado laboral, brecha que se encuentra entre los 10-12 puntos porcentuales. Ello provoca una mayor pobreza en las mujeres como consecuencia de su no incorporación al mercado laboral.

Tabla 1.

Tasa de actividad en el periodo 2012-2021. Tercer trimestre. Cifras en porcentaje. Fuente: Elaboración propia en base a INE (2021)

\begin{tabular}{lccc}
\hline & TOTAL & Hombres & Mujeres \\
\hline 3T 2012 & 60,55 & 67,37 & 54,02 \\
3T 2013 & 60,04 & 66,61 & 53,78 \\
3T 2014 & 59,53 & 66,02 & 53,35 \\
3T 2015 & 59,50 & 65,90 & 53,42 \\
3T 2016 & 59,28 & 65,26 & 53,61 \\
3T 2017 & 58,92 & 65,04 & 53,13 \\
3T 2018 & 58,73 & 64,86 & 52,93 \\
3T 2019 & 58,72 & 64,46 & 53,28 \\
3T 2020 & 57,83 & 63,44 & 52,53 \\
3T 2021 & 59,14 & 64,31 & 54,25 \\
\hline
\end{tabular}

En la tabla 1 se muestra como en el periodo temporal analizado, la incorporación de la mujer al mundo laboral, considerada a través de la población activa, es mucho menor que la del hombre, con una media de 10 puntos porcentuales. Este resultado muestra la dificultad que tiene la mujer para incorporarse al mercado laboral, en ocasiones derivada de su necesidad de cuidar de los hijos 0 de personas dependientes, tal y como señala la literatura científica previa (DomínguezOlabide, 2021; Quintero Lima y Díaz Gorkinkiel, 2021), o debido al tipo de formación que tiene la mujer, tal y como corrobora la literatura científica (Choi, 2021).

\subsection{Análisis de la tasa de paro. Periodo 2012-2021}

La tasa de paro viene dada por la relación entre la población desempleada y la población activa. En la tabla 2 se muestra la tasa de paro en España en los diez últimos años, correspondientes a los datos de la EPA del tercer trimestre de cada año. Se utiliza los datos del tercer trimestre para poder realizar una comparación entre dichos datos, correspondientes al mismo periodo temporal.

En dicha tabla se muestra claramente como existe una brecha de género en cuanto a los problemas que tiene la mujer para incorporarse al mercado laboral. De hecho, en los últimos años esta brecha se encuentra situada entre 3-4 puntos porcentuales, siendo en todos los años la tasa de paro mayor en las mujeres que en los hombres. Esta situación provoca unos mayores niveles de pobreza en las mujeres como consecuencia de la dificultad que tiene las mujeres para acceder a un puesto de trabajo. 
Tabla 2.

Tabla de paro en el periodo 2012-2021. Tercer trimestre. Cifras en porcentaje. Fuente: Elaboración propia en base a INE (2021)

\begin{tabular}{cccc}
\hline & TOTAL & Hombres & Mujeres \\
\hline 3T 2012 & 24,79 & 24,54 & 25,10 \\
3T 2013 & 25,65 & 25,19 & 26,18 \\
3T 2014 & 23,67 & 22,53 & 25,01 \\
3T 2015 & 21,18 & 19,90 & 22,69 \\
3T 2016 & 18,91 & 17,39 & 20,66 \\
3T 2017 & 16,38 & 14,80 & 18,21 \\
3T 2018 & 14,55 & 13,12 & 16,22 \\
3T 2019 & 13,92 & 12,17 & 15,92 \\
3T 2020 & 16,26 & 14,39 & 18,39 \\
3T 2021 & 14,57 & 12,97 & 16,36 \\
\hline
\end{tabular}

En la tabla 2 se muestra de forma clara la brecha existente en cuanto a la incorporación plena de la mujer al mundo laboral. Así, y siguiendo a Aragón Gómez (2018), es necesario establecer en España políticas laborales que posibiliten la incorporación de la mujer al mundo laboral con el objetivo de reducir la brecha que actualmente existe. Además, esta situación ha empeorado con la pandemia de la Covid-19 debido a que ha tenido un mayor impacto en el sector servicios y, como señala Albanesi y Kim (2021), un sector más feminizado y donde la mujer ocupa los puestos de trabajo más vulnerables a la evolución cíclica de la economía.

\subsection{Análisis de la situación de los cuidados de hijos y de personas dependientes}

Uno de los grandes problemas que tienen las mujeres para incorporase al mercado de trabajo es el cuidado de los hijos y de personas dependientes, tarea que recae mayoritariamente sobre las mujeres. A su vez, esta situación deriva del hecho de que muchas familias no pueden costear la ayuda de otras personas para realizar este tipo de tareas y que, finalmente, recaen sobre la mujer. Ello provoca que, el colectivo de las mujeres pasa a ser, en muchas ocasiones, población inactiva, sin que puedan disponer de recursos económicos, lo que acarrea, incluso, no solo una pobreza de dicho colectivo sino de la propia familia en sí, en especial, cuando se tratan de familias monoparentales.

En la tabla 3 se muestra las razones por las cuáles las personas se dedican al cuidado de los hijos y de las personas dependientes en el periodo temporal 2016-2020.

La tabla 3 muestra como un porcentaje de mujeres tienen que abandonar el mercado laboral para cuidar de sus hijos pequeños o de las personas dependientes. Esta clase de trabajo, muchas veces desconocido para la sociedad, implica el paso de las mujeres a la población inactiva o a mercados de trabajo informales. Los resultados obtenidos refrendan las aportaciones previas de la literatura científica (Pérez Corral y Moreno Mínguez, 2021) donde se aborda cómo este tipo de trabajo, tan fundamental en una sociedad avanzada, puede implicar una brecha de género aún mayor en la pobreza de las mujeres, sobre todo, cuando se trata de familias monoparentales. 
Tabla 3.

Tasa de personas que se dedican al cuidado de personas dependientes y de niños. Periodo 20162020. Cifras en porcentaje. Fuente: Elaboración propia en base a INE (2021)

\begin{tabular}{lccccc}
\hline & $\mathbf{2 0 1 6}$ & $\mathbf{2 0 1 7}$ & $\mathbf{2 0 1 8}$ & $\mathbf{2 0 1 9}$ & $\mathbf{2 0 2 0}$ \\
\hline $\begin{array}{l}\text { Ambos sexos } \\
\text { Total }\end{array}$ & 100 & 100 & 100 & 100 & 100 \\
$\begin{array}{l}\text { Por no haber o no poder costear los servicios } \\
\text { adecuados para el cuidado de niños }\end{array}$ & 39,5 & 39,6 & 34,2 & 31,7 & 35,8 \\
$\begin{array}{l}\text { Por no haber o no poder costear los servicios } \\
\text { adecuados para el cuidado de adultos enfermos }\end{array}$ & 21,1 & 19,9 & 16,9 & 20,1 & 18,4 \\
Por ambos motivos & 4,0 & 3,5 & 4,1 & 4,4 & 4,2 \\
Por otros motivos diferente & 33,7 & 36,5 & 43,6 & 43,1 & 41,1 \\
No sabe & 1,7 & 0,5 & 1,2 & 0,7 & 0,4 \\
HOMBRES & & & & & \\
Total & 100 & 100 & 100 & 100 & 100 \\
Por no haber o no poder costear los servicios & 8,4 & 13,9 & 9,0 & 8,3 & 13,3 \\
adecuados para el cuidado de niños & & & & & \\
Por no haber o no poder costear los servicios & 46,8 & 44,5 & 48,5 & 58,4 & 34,0 \\
adecuados para el cuidado de adultos enfermos & 3,5 & 0,0 & 5,2 & 1,0 & 0,3 \\
Por ambos motivos & 31,4 & 41,6 & 35,5 & 31,6 & 52,4 \\
Por otros motivos diferente & 0,0 & 0,0 & 1,8 & 0,6 & 0,0 \\
No sabe & & & & & \\
MUJERES & 100 & 100 & 100 & 100 & 100 \\
Total & 41,4 & 41,2 & 35,8 & 33,5 & 37,7 \\
Por no haber o no poder costear los servicios & 18,9 & 18,3 & 15,0 & 17,2 & 17,2 \\
adecuados para el cuidado de niños & 4,1 & 3,8 & 4,1 & 4,6 & 4,5 \\
Por no haber o no poder costear los servicios & 33,8 & 36,2 & 44,1 & 44,0 & 40,2 \\
adecuados para el cuidado de adultos enfermos & 1,8 & 0,6 & 1,1 & 0,7 & 0,5 \\
\hline $\begin{array}{l}\text { Por ambos motivos } \\
\text { Por otros motivos diferente }\end{array}$ & & & & & \\
No sabe & & & & \\
\hline
\end{tabular}

4.4. Análisis de la relación entre la tasa de actividad y la tasa de paro. Brecha de género tercer trimestre de 2021

Cuando se analiza el mercado de trabajo, es necesario estudiar al mismo tiempo dos tasas como serían la tasa de paro y la tasa de actividad. De hecho, y aunque los datos de estas dos tasas se suelen presentar de forma totalmente independiente, estas tasas, y los resultados que se extraen de ellas, están completamente relacionados.

En este apartado de la sección de Resultados se plantea un análisis empírico consistente en considerar que la tasa de actividad entre el hombre y la mujer es la misma. Es decir, qué ocurriría con la tasa de paro si la población activa de ambos géneros es la misma, con lo cual la tasa de actividad también sería la misma. En consecuencia, la pregunta de investigación a responder sería la siguiente: si la tasa de actividad es la misma entre mujeres y hombres, cuál sería realmente la tasa de paro de las mujeres. Para realizar este estudio empírico se van a utilizar los datos de la EPA correspondientes al tercer trimestre de 2021.

En la tabla 4 se recogen los datos de la EPA del tercer trimestre de 2021 correspondientes a la tasa de actividad y a la tasa de paro diferenciada por género. 
Tabla 4.

Tabla de actividad y tasa de paro. Tercer trimestre de 2021. Cifras en porcentaje. Fuente: Elaboración propia en base a INE (2021)

\begin{tabular}{lccc}
\hline \multicolumn{1}{c}{ EPA } & TOTAL & HOMBRES & MUJERES \\
\hline Tasa de actividad & 59,14 & 64,31 & 54,25 \\
Tasa de paro & 14,57 & 12,97 & 16,36 \\
\hline
\end{tabular}

De acuerdo con los datos de la EPA del tercer trimestre de 2021, la tasa de actividad de las mujeres es del $54,25 \%$. Ello implica que, de cada 100 mujeres en edad de trabajar, 54,25 personas quieren trabajar.

En cuanto a la tasa de paro de las mujeres, implicaría que de cada 54,25 mujeres que quieren trabajar, 16,36 mujeres no encuentran trabajo. Es decir, ello implicaría que de cada 54,25 mujeres que quieren trabajar 9,779 mujeres no encuentran trabajo.

Por tanto,

Tasa de paro $=\left(\frac{\text { población desempleada }}{\text { población activa }}\right) \times 100=>$ Población desempleada $=$ población activa $\times$ tasa de paro $=54,25 \times 0,1636=8,87$

Si consideramos que la tasa de actividad pasaría del $54,25 \%$ al $64,31 \%$, ello implicaría que 10,06 mujeres más de cada 100 se querían incorporar al mundo laboral, aunque estas mujeres no encontrarían trabajo ya que el número de mujeres ocupadas seguirían siendo el mismo. En consecuencia, aumentaría la tasa de paro de las mujeres ya que estas 10,06 mujeres más de cada 100 que pasaran de población inactiva a población activa entrarían como desempleadas.

Para saber cuál sería la tasa de paro en esta situación, deberíamos de tener en cuenta la nueva población activa $(64,31)$, coincidente con la tasa de población activa de los hombres, y el número actual de mujeres desempleadas $(8,87+10,06=18,93)$. Por tanto:

$$
\text { Tasa de paro }=\left(\frac{\text { población desempleada }}{\text { población activa }}\right) \times 100=\left(\frac{18,93}{64,31}\right) \times 100=29,44 \%
$$

En conclusión, el número de personas desempleadas está muy relacionado tanto con la tasa de actividad como con la tasa de paro. Ello implica que si la tasa de actividad entre hombres y mujeres fuera similar, y no variase el número de mujeres ocupadas, la tasa de paro femenina sería mucho más elevada que lo es en actualidad, y tendría una brecha aún mayor entre hombres y mujeres. En este estudio empírico el resultado sería de una diferencia de más de 16 puntos porcentuales $(29,44 \%$ - $12,97 \%)$ entre los hombres y las mujeres, resultado que reforzaría la feminización de la pobreza, y que implicaría la necesidad de realizar políticas laborales y de conciliación para facilitar y consolidar el acceso de la mujer al mundo laboral.

\section{Conclusiones}

Los niveles de pobreza de las mujeres en España han ido incrementándose de forma significativa con el paso del tiempo, especialmente debido a las condiciones laborales. Así, se deriva de la dificultad tanto del acceso al mercado laboral como de la permanencia en el mismo para las mujeres. Todo ello ha ocasionado un proceso consolidado de feminización de la pobreza en España, que se ha agudizado con las dos crisis económicas de los años 2008 y 2020 . De hecho, las crisis económicas ponen de relieve las desigualdades que sufren las mujeres e intensifican aún más las situaciones de pobreza de este colectivo. Esta conclusión muestra la 
necesidad de que desde las distintas administraciones públicas se realizan políticas laborales que apuesten por la mujer y su incorporación al mercado laboral en condiciones dignas.

Los principales resultados de esta investigación muestran como existe una fuerte brecha entre la población activa y la población inactiva por género en España. De hecho, esta brecha es de aproximadamente 16 puntos porcentuales en los últimos años. Por esta razón, es necesaria la instauración de políticas que permita una incorporación real de la mujer al mundo laboral, sobre todo para que le permita una mayor conciliación con la vida familiar.

Por otro lado, otro de los resultados obtenidos en esta investigación es cómo la mujer que sí se incorpora al mundo laboral tiene unas tasas de paro muy superiores a la de los hombres en los últimos años. Estos datos refuerzan la necesidad de adaptar los niveles formativos de las mujeres a un mercado laboral cada vez más cambiante, y que les dé acceso a estos nuevos puestos de trabajo.

Un tercer resultado procedente de un análisis empírico donde la tasa de paro de la mujer sería mucho mayor al que dicta los datos oficiales si realmente la incorporación de la mujer fuese la misma que la del hombre, es decir, tuviese una misma tasa de actividad. Así, en este supuesto, y de acuerdo con los datos indicados en esta investigación, la brecha sería de unos 16 puntos porcentuales.

La principal aplicación práctica de esta investigación es conocer la situación actual del mercado laboral en España y mostrar algunas soluciones para que las diferentes administraciones públicas puedan diseñar políticas que favorezca a la mujer.

La principal limitación de esta investigación se encuentra en que el periodo temporal analizado (2012-2021) no recoge la salida de la crisis provocada por la pandemia de la Covid-19. Como futura línea de investigación, se recomienda reforzar las investigaciones que aborden cuál ha sido para las mujeres el impacto de la salida de la Crisis de 2008 y cuál va a ser para las mujeres el impacto de la salida de la Crisis de 2020.

\section{Referencias}

Albanesi, S. y Kim, J. (2021). Effects of the COVI-19 recession on the US labor market: Occupation, family, and gender. Journal of Economic Perspectives, 35(3), 3-24.

Aragón Gómez, C. (2018). Mujer y acceso al mercado de trabajo. Revista Internacional y Comparada de Relaciones Laborales y Derecho del Empleo, 6(4), 45-69.

Choi, A. (2021). España ante la Revolución Industrial 4.0: mercado laboral y formación. Araucaria. Revista Iberoamericana de Filosofía, Política, Humanidades y Relaciones Laborales, 47, 479-505.

Cebrián López, I. y Llorente Heras, R. (2017). Mujer y mercado de trabajo. Revista de Economía Laboral, 14(2), 1-4.

Cortès-Franch, I. y Artazcoz, L. (2021). Desigualdades de género relacionadas con la flexibilidad laboral. Archivos de Prevención de Riesgos Laborales, 24(4), 335-341.

Dios-Murcia, I., Hospido, L., Pena-Boquete, Y. y Román, C. (2021). Trabajo en plataformas digitales: ¿Una oportunidad para la oferta laboral femenina? Boletín Económico de ICE, Información Comercial Española, 921, 101-125.

Domínguez-Olabide, P. (2021). Prestaciones para la conciliación de la vida laboral y familiar: una revisión de su diseño e impacto en los países de la OCDE. Zerbitzuan, 74, 33-54.

Gómez Rufián, L. (2018). La penalización de la mujer en el mercado de trabajo desde la perspectiva de las transiciones laborales. Revista de Información Laboral, 4, 225-244. 
Iglesias Fernández, C. y Llorente Heras, R. (2001). Mujer y mercado de trabajo. Una revisión de la situación actual para el caso de la Comunidad de Madrid. Revista de Estudios Regional, 61, 15-42.

López Rodríguez, A. A. y González Gómez, A. (2021). IV Revolución industrial y mercado laboral en España: un escenario post covid-19. Revista de Investigaciones Políticas y Sociológicas, 20(1), 34-57.

Moreno Márquez, A. M. (2021). Género, formación profesional e inserción laboral. Asparkía, 38, 83-104.

Pérez Corral, A. L. y Moreno Mínguez, A. (2021). Estructura familiar, deterioro del mercado laboral y desigualdad en España para el período 2008-2017. Revista Internacional de Sociología, 79(2). e186. https://doi.org/10.3989/ris.2021-79.2.19.131.

Quintero Lima, M. y Díez Gorfinkiel, M. (2021). Los cuidados, nuevos mercados y el trabajo de las mujeres: de la necesidad de nuevas perspectivas económicas. Boletín Económico de ICE, Información Comercial Española, 921, 51-64.

Reichelt, M., Makovi, K. y Sargsyan, A. (2021). The impact of COVI-19 on gender inequality in the labor market and gender-role attitudes. European Societies, 23(S1), S228-S-245. https://doi.org/10.1080/14616696.2020.1823010

Salido Cortés, L. (2021). Los efectos de la pandemia sobre la igualdad de género: algunos análisis sobre el mercado de trabajo español. Panorama Social, 33, 75-93. 